\title{
Eosinophil cationic protein: A new diagnostic biomarker in coronary slow flow phenomenon
}

\author{
Soylu $\mathrm{K}^{1}$, Akcay $\mathrm{M}^{1}$, Aksan $\mathrm{G}^{2}$, Gedikli $\mathrm{O}^{1}$, Gokay $\mathrm{N}^{3}$ \\ Department of Cardiology, Faculty of Medicine, OndokuzMayisUniversity, Samsun, Turkey. \\ drmuratakcay@hotmail.com
}

\begin{abstract}
AIM: This study has investigated the role of eosinophil cationic protein (ECP), released by eosinophils, in the coronary slow flow phenomenon.

METHODS: This study included sixty patients with coronary slow flow (CSF) and sixty patients with normal coronary flow. The coronary flow rate was evaluated with TIMI frame count (TFC). ECP level, blood count and biochemical parameters were assessed.

RESULTS: The ECP levels $(18.9 \pm 7.5$ vs $13.1 \pm 6.4 \mathrm{ng} / \mathrm{ml}, p<0.001)$ and eosinophil counts $(0.25 \pm 0.14$ vs $\left.0.18 \pm 0.0910^{3} / \mathrm{mm}^{3}, p=0.001\right)$ were higher in the CSF group. Multivariable regression analysis showed that ECP level and eosinophil counts were independent predictors the presence of CSF $(p=0.003$ and $p=0.006)$. There was a weak but important correlation among the ECP level, eosinophil count and mean TFC $(p=0.001$, $p=0.003$, respectively). The ROC analysis showed a cut off value of $14.05 \mathrm{ng} / \mathrm{ml}$ for ECP level to diagnose CSF with $73.3 \%$ sensitivity and $66.7 \%$ specificity, and area under the ROC curve was $0.745(95 \% \mathrm{Cl}$ : $0.657-0.833, p<0.001)$.

CONCLUSION: ECP levels were increased in CSF patients and this increasing correlated with coronary artery flow rates. The ECP level was independent predictor for the presence of SCF and it may be use as suitable diagnostic biomarker for CSF (Tab. 3, Fig. 3, Ref. 30). Text in PDF www.elis.sk KEY WORDS: coronary slow flow phenomenon, eosinophil cationic protein, eosinophil.
\end{abstract}

\section{Introduction}

Coronary slow flow phenomenon (CSFP) is described as an abnormal reduction in coronary artery flow rate with no concomitant epicardial stenosis to block the flow. While it is usually of benign course, it is reported to be related with severe cardiovascular events in some cases (1-2). Studies mostly support the link between CSF and microvascular dysfunction. In addition, endothelial dysfunction, atherosclerosis, platelet and red cell dysfunction have also been shown in these patients (1-3).

Eosinophils are also involved in endothelial dysfunction, inflammation and thrombosis with secreting some allergic inflammatory, vasoactive and pro-coagulant mediators (3-4). While the role of neutrophils in atherosclerosis has been well investigated, there are few reports on increased levels of eosinophils in coronary artery disease. Eosinophilic inflammation has been associated with the pathogenesis of coronary dissection, stent restenosis and coronary artery ectasia (4). A study performed at our clinic has revealed increased eosinophil count in patients diagnosed with coronary slow flow phenomenon (4-5). Additionally, there was an important correlation among TIMI (Thrombolysis in Myocar-

Ondokuz Mayis University, Faculty of Medicine, Department of Cardiology, Samsun, Turkey

Address for correspondence: Akcay M, MD, Ondokuz Mayis University, Faculty of Medicine, Department of Cardiology, 55139 Samsun, Turkey. Phone: +90.506 .7795760$ dial Infarction) frame count (TFC) values and eosinophil counts. However, no eosinophilic marker has been analyzed.

In here, we aimed to investigate the role of eosinophil cationic protein (ECP), released by eosinophils and called an eosinophiloriginated inflammatory mediator, in the coronary slow flow phenomenon.

\section{Methods}

\section{Study population}

The study was planned as a cross-sectional randomized study. A total of 120 patients were included in this study; sixty patients with coronary slow flow (CSF) and sixty patients with normal coronary flow (NCF) were randomly selected from angiographic records. Patients with acute coronary syndrome, coronary artery ectasia, left ventricular systolic dysfunction $(\mathrm{EF}<55 \%)$, significant valvular disease, chronic renal disease, coronary plaque in any coronary artery, previous myocardial infarction, percutaneous coronary intervention or coronary artery bypass grafting, noncoronary atherosclerosis, autoimmune disease, malignancy, rheumatologic disease, thyroid disease, active parasitic disease, history of asthma, atopic dermatitis and other allergic diseases were excluded from the study.

The study protocol and patient's informed consent statements were signed by patients and followed the ethical guidelines described by the Declaration of Helsinki. 


\section{Coronary angiography}

All patients underwent coronary angiography using the Judkins method and femoral way. The data was recorded during the procedure using digital angiographic system (ACOM.PC; Siemens AG, Germany) at rate of 15 frame/sec. The iopromide (Ultravist 370, Schering AG, Berlin, Germany) was used as the contrast material. Gibson et al study (6) was taken as reference and the TFC values were evaluated as in that study. The angiographic images were examined by two independent cardiologists for each coronary artery. In Gibson et al (6) study, the recording rate was $30 \mathrm{frame} / \mathrm{sec}$, so in our study, the detected values were multiplied by two. When differences were detected between the measurements, the analyses were repeated by two cardiologists and mean values were taken. Finally, since the left anterior descending (LAD) artery was longer, the TFC values were divided by 1.7 , for LAD and corrected TFC (cTFC) values were obtained (5-6).

\section{Definition of coronary slow flow (CSF)}

Gibson et al detected the mean TFC value of normal coronary arteries as $36.2 \pm$ 2.6 for LAD, $22.2 \pm 4.1$ for left circumflex artery (LCx), and $20.4 \pm 3$ for right coronary artery (RCA) (6). Thus, a TFC value detected to be two standard deflections above the average value suggests the existence of slow flow in the relevant coronary artery. Therefore, detection of $\geq 42$ for $\mathrm{LAD}, \geq 31$ for LCx and $\geq 27$ values for RCA was accepted as CSF, in our study (7).

\section{Laboratory measurements}

The blood was taken in a sitting position from the antecubital vein. All blood tests were taken with fasting, between 8-10 am, to avoid diurnal changes. All routine biochemical and hematologic parameter measurements were obtained on the day of blood draw. All biochemical and hematologic parameters were analyzed using with Abbott ARCHITECT c 8000 (Abbott Laboratories, USA) auto-analyzer with commercial kits and Abbott Cell Dyn 3700 (Abbott Laboratories, USA) device on the same day.

Serum Eosinophil Cationic Protein (ECP) levels were determined using a Triturus ${ }^{\circledR}$ enzyme-linked immunosorbent assay (ELISA) analyzer (Grifols International, Barcelona, Spain) device with Human Eosinophil Cationic Protein (ECP) commercial kit (Cusabio Biotech Company, PRC), according to the manufacturer's instructions. A standard curve was formed with a four- parameter logistic curve-fit (4-PL) measurement technique and the outcomes were measured as $\mathrm{ng} / \mathrm{ml}$ based on this curve.

\section{Statistical analysis}

The research data were transferred to a computer and analyzed using IBM SPSS Statistics for Windows, Version 20.0 (IBM Corp., Armonk, NY, USA). Descriptive statistics were expressed as mean, standard deviation, frequency and percentage. The KolmogorovSmirnov test was used to evaluate whether the continuous variables

Tab. 1. Baseline demographic characteristics, echocardiographic and laboratory parameters and risk factors of patients.

\begin{tabular}{|c|c|c|c|}
\hline Variable & $\begin{array}{c}\text { Coronary slow } \\
\text { flow }(n=60)\end{array}$ & $\begin{array}{c}\text { Normal coronary } \\
\text { flow }(n=60)\end{array}$ & $\mathrm{p}$ \\
\hline \multicolumn{4}{|l|}{ Clinical and hemodynamic data } \\
\hline Age (years) & $56.4 \pm 10.3$ & $57.0 \pm 10.1$ & 0.73 \\
\hline Men, n (\%) & $41(68.3)$ & $37(61.7)$ & 0.44 \\
\hline Women, n (\%) & $19(31.7)$ & $23(38.3)$ & \\
\hline Hypertension, $\mathrm{n}(\%)$ & $49(81.7)$ & $42(70.0)$ & 0.14 \\
\hline Diabetes mellitus, n (\%) & $16(26.7)$ & $14(23.3)$ & 0.67 \\
\hline Smoking, n (\%) & $28(46.7)$ & $20(33.3)$ & 0.14 \\
\hline Heart rate (beat/min) & $78.1 \pm 9.7$ & $75.1 \pm 10.9$ & 0.11 \\
\hline Systolic blood pressure (mmHg) & $126.3 \pm 17.1$ & $126.6 \pm 15.7$ & 0.62 \\
\hline Diastolic blood pressure $(\mathrm{mmHg})$ & $77.5 \pm 10.3$ & $76.6 \pm 10.2$ & 0.93 \\
\hline Left Ventricular Ejection fraction, \% & $61.9 \pm 4.8$ & $63.3 \pm 3.7$ & 0.10 \\
\hline \multicolumn{4}{|l|}{ Drug use, $\mathrm{n}(\%)$} \\
\hline ACEI/ARB & $28(46.7)$ & $24(40)$ & 0.46 \\
\hline $\mathrm{CCB}$ & $6(10)$ & $8(13.3)$ & 0.57 \\
\hline DU & $17(28.3)$ & $16(26.7)$ & 0.84 \\
\hline Statin & $12(20)$ & $14(23.3)$ & 0.66 \\
\hline \multicolumn{4}{|l|}{ Biochemical and hematological data } \\
\hline Total cholesterol, mg/dl & $223.2 \pm 50.1$ & $188.1 \pm 32.5$ & $<0.001$ \\
\hline Low-density lipoprotein (LDL), mg/dl & $138.5 \pm 35.7$ & $111.7 \pm 25.7$ & $<0.001$ \\
\hline High-density lipoprotein (HDL), mg/dl & $36.5 \pm 8.6$ & $46.6 \pm 8.9$ & $<0.001$ \\
\hline Triglyceride, $\mathrm{mg} / \mathrm{dl}$ & $206.1 \pm 119.3$ & $152.2 \pm 81.8$ & 0.005 \\
\hline Creatinine, $\mathrm{mg} / \mathrm{dl}$ & $0.82 \pm 0.17$ & $0.76 \pm 0.19$ & 0.11 \\
\hline Fasting glucose, mg/dl & $105.9 \pm 21.2$ & $98.7 \pm 36.9$ & 0.21 \\
\hline \multicolumn{4}{|l|}{ Corrected TIMI frame count } \\
\hline Left anterior descending artery & $38.8 \pm 10.8$ & $20.0 \pm 8.0$ & $<0.001$ \\
\hline Circumflex coronary artery & $47.9 \pm 32.1$ & $18.3 \pm 5.6$ & $<0.001$ \\
\hline Right coronary artery & $55.8 \pm 30.5$ & $20.9 \pm 7.4$ & $<0.001$ \\
\hline Mean TFC & $47.5 \pm 19.4$ & $19.7 \pm 5.1$ & $<0.001$ \\
\hline \multicolumn{4}{|l|}{ Slow flow related coronary artery, $\mathrm{n}(\%)$} \\
\hline Left anterior descending artery & $37(61.7)$ & & \\
\hline Circumflex coronary artery & $41(68.3)$ & & \\
\hline Right coronary artery & $48(80)$ & & \\
\hline Single coronary slow flow & $20(33.3)$ & & \\
\hline Multiple coronary slow flow & $40(66.6)$ & & \\
\hline
\end{tabular}

ACEI - Angiotensin-converting enzyme inhibitors, ARB - Angiotensin receptor blockers, CCB - Calcium channel blockers, DU - Diuretic drug

Tab. 2. Basic haematological parameters of coronary slow flow and normal flow patients.

\begin{tabular}{lccc}
\hline Variable & $\begin{array}{c}\text { Coronary slow } \\
\text { flow }(\mathrm{n}=60)\end{array}$ & $\begin{array}{c}\text { Normal coronary } \\
\text { flow }(\mathrm{n}=60)\end{array}$ & $\mathrm{p}$ \\
\hline Hemoglobin, gr/dl & $14.4 \pm 1.4$ & $14.2 \pm 1.6$ & 0.52 \\
Platelet, $10^{3} / \mathrm{mm}^{3}$ & $264.3 \pm 66.6$ & $253.3 \pm 52.9$ & 0.32 \\
White blood cell count, $10^{3} / \mathrm{mm}^{3}$ & $7.27 \pm 2.49$ & $7.23 \pm 1.84$ & 0.93 \\
Neutrophil, $10^{3} / \mathrm{mm}^{3}$ & $4.10 \pm 1.82$ & $3.98 \pm 1.25$ & 0.66 \\
Lymphocyte, $10^{3} / \mathrm{mm}^{3}$ & $2.15 \pm 6.31$ & $2.29 \pm 6.03$ & 0.21 \\
Basophil, $10^{3} / \mathrm{mm}^{3}$ & $0.065 \pm 0.02$ & $0.068 \pm 0.02$ & 0.47 \\
Eosinophil, $10^{3} / \mathrm{mm}^{3}$ & $0.25 \pm 0.14$ & $0.18 \pm 0.09$ & 0.001 \\
ECP, $\mathrm{ng} / \mathrm{ml}$ & $18.9 \pm 7.5$ & $13.1 \pm 6.4$ & $<0.001$ \\
\hline
\end{tabular}


Tab. 3. Logistic regression analysis to determine the independent predictors of coronary slow flow.

\begin{tabular}{|c|c|c|c|c|c|c|}
\hline \multirow[t]{2}{*}{ Predict } & \multicolumn{3}{|c|}{$\begin{array}{c}\text { Univariate logistic regression } \\
\text { analysis }\end{array}$} & \multicolumn{3}{|c|}{$\begin{array}{c}\text { Multivariate logistic regression } \\
\text { analysis }\end{array}$} \\
\hline & Odds Ratio & $95 \% \mathrm{CI}$ & $\mathrm{p}$ & Odds Ratio & $95 \% \mathrm{CI}$ & $\mathrm{p}$ \\
\hline Men & 0.745 & $0.351-1.583$ & 0.44 & & & \\
\hline Age, years & 1.006 & $0.971-1.043$ & 0.72 & & & \\
\hline $\mathrm{ECP}(\mathrm{ng} / \mathrm{ml})$ & 1.135 & $1.065-1.209$ & $<0.001$ & 1.106 & $1.034-1.183$ & 0.003 \\
\hline Eosinophil, $10^{3} / \mathrm{mm}^{3}$ & 1.006 & $1.002-1.009$ & 0.002 & 1.006 & $1.002-1.010$ & 0.006 \\
\hline Fasting glucose (mg/dl) & 1.008 & $0.996-1.020$ & 0.21 & & & \\
\hline Creatinine & 5.358 & $0.683-42.033$ & 0.11 & & & \\
\hline $\mathrm{LVEF} \%$ & 0.931 & $0.854-1.014$ & 0.10 & & & \\
\hline LDL (mg/dl) & 1.030 & $1.015-1.045$ & $<0.001$ & 1.034 & $1.016-1.052$ & $<0.001$ \\
\hline Smoking & 1.750 & $0.836-3.662$ & 0.14 & & & \\
\hline
\end{tabular}

Low-Density Lipoprotein and smoking). Inclusion of variables into the final models was based on both clinical and statistical conditions. Analyses were repeated using forward stepwise analysis to control the consistency of the results. The final model was able to correctly predict $76.3 \%$ of patients with CSF. The diagnostic power of ECP in CSF was evaluated by receiver operating characteristic (ROC) curve. A probability value $<0.05$ was considered the minimum value of statistical importance. A two-sided p-value was used for all comparisons.

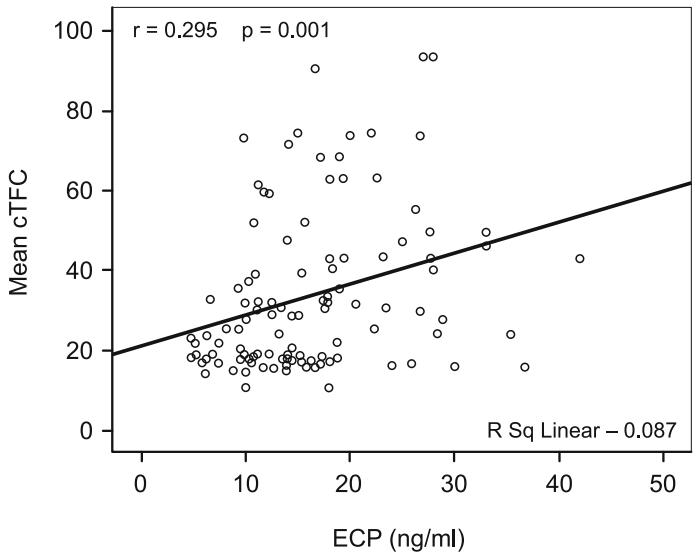

Fig. 1. Correlation between eosinophil cationic protein level (ECP) and the mean corrected TIMI frame count.

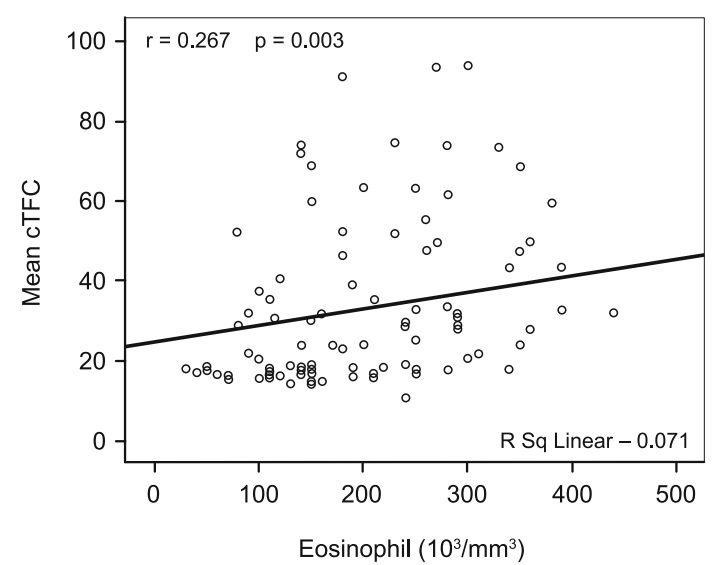

Fig. 2. Correlation between eosinophil count and the mean corrected TIMI frame count.

were normally distributed. For continuous variables, the independent samples t-test was used. Any correlation among variables was evaluated with Pearson's correlation analysis. Multivariate logisticregression analysis was also performed and the model included the potential confounders (Age, Sex, Eosinophil, ECP, Fasting Glucose, Serum Creatinine, Left Ventricular Ejection Fraction,

\section{Results}

The study included sixty CSF and sixty NCF patients. The patients' basic demographic characteristics, clinical and laboratory parameters are presented in Table 1 . The mean TFC level was prominently higher in the CSF group relative to the NCF group ( $47.5 \pm 19.4$ versus $19.7 \pm 5.1 ; \mathrm{p}<0.001)$. In addition, the lipid parameters were markedly higher in the CSF group $(\mathrm{p}<0.001)$. In the CSF group, 37 (61.7\%), 41 (68.3\%) and 48 patients (80\%) had CSF in the LAD artery, LCx artery and RCA artery, respectively. While 40 patients $(66.6 \%)$ had CSF in two or three vessels, 20 patients $(33.3 \%)$ had CSF in a single coronary artery.

Comparison of the blood cell counts between groups showed that WBC and neutrophil counts were numerically higher in the CSF group, but not statistically important $(\mathrm{p}>0.05)$. In contrast, lymphocyte and basophil counts were numerically lower and statistically insignificant $(\mathrm{p}>0.05)$. The eosinophil count was markedly higher in the CSF group ( $\mathrm{p}=0.001)$. In addition, the ECP level was importantly higher in the CSF group $(p<0.001)$ (Tab. 2). There was a weak but important correlation between the ECP level and mean TFC $(r=0.295, p=0.001)$ (Fig. 1). In addition, eosinophil count also showed a weak but important correlation with mean TFC ( $r=0.267, p=0.003)$ (Fig. 2). In the multivariate regression analysis, the ECP level $(p=0.003)$, eosinophil count $(p=0.006)$, and LDL levels $(p<0.001)$ were independent predictor variables for the presence of CSF (Tab. 3). We furthermore performed receiver operating characteristic (ROC) analysis to detect whether ECP level can serve as an independent diagnostic indicator of CSF. The ROC analysis showed a cut off value of $14.05 \mathrm{ng} / \mathrm{ml}$ for ECP level to diagnose CSF with $73.3 \%$ sensitivity and $66.7 \%$ specificity, and the area under the ROC curve (AUC) was 0.745 (95\% CI: 0.657-0.833, p <0.001) (Fig. 3).

\section{Discussion}

In this study, we found that patients with CSF phenomenon had increased eosinophil counts and ECP levels, which also correlated with the coronary flow rate. In addition, plasma ECP level showed a statically important diagnostic power in patients with CSF. This is the first study to evaluate the eosinophilic cationic protein level in patients with CSF phenomenon. 


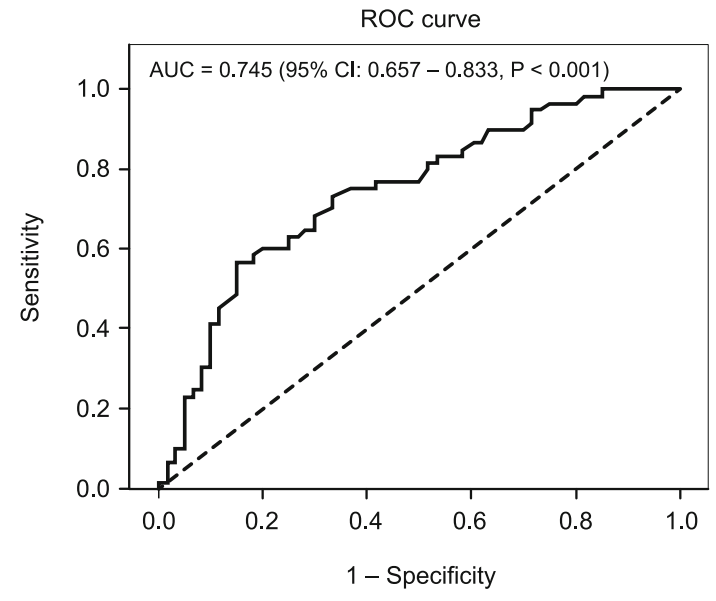

Fig. 3. Analysis of receiver operating characteristic (ROC) to determine whether ECP level can serve as an independent diagnostic indicator of CSF.

Previous studies suggest that microvascular and endothelial dysfunction, diffuse atherosclerosis, platelets, erythrocytes and vasomotor dysfunction may be involved in the CSF etiology (2, $5,7-10)$. In contrast, inflammation is deemed as another significant factor involved in CSF phenomenon development. Increased neutrophil activation has been demonstrated particularly in patients with CSF as well as those with atherosclerotic coronary artery disease (8). Li et al (9) demonstrated that inflammatory mediators such as C-reactive protein and interleukin- 6 values were importantly raised in the CSF patients. Turhan et al (10) found that inflammatory mediators such as plasma ICAM-1, VCAM-1, and E-selectin values were higher in the CSF patients and positively correlated with TFC. However, studies have been focused on the role of allergic inflammation in coronary events. Eosinophils and the activation indicator mediators of eosinophils such as ECP, major basic protein, eotaxin are increasingly used for sensitive monitoring of allergic inflammation (3).

Eosinophils may lead to vascular injury by triggering the coagulation system and the platelet activation (11). While the active role of eosinophils has been demonstrated in allergic and atopic disorders, it's role in coronary artery disease has not been sufficiently investigated. Umemotoet et al (12) found markedly higher eosinophil counts in patients with severe vasospastic angina. The hypersensitivity reaction triggered by eosinophils may also lead to Kounis syndrome, which has variations including allergic myocardial infarction and vasospastic allergic angina (13). In both studies, increased eosinophil counts were seen similarly in patients with coronary artery disease (14). In addition, histopathological studies conducted in patients with acute coronary syndrome revealed increased eosinophilic infiltrates in coronary thrombus specimens (15).

Eosinophil granule proteins may result in strong prothrombotic activity via platelet activation (16-17). While the major basic protein, ECP, and eosinophil originated neurotoxin are the primary eosinophilic mediators related with human tissue injury, these have also been associated with eosinophilic myocarditis, pneumonitis, dermatitis, neuropathy and vasculitis development (11). On the other hand, there is restricted data on the role of granule proteins, indicating eosinophil activation in coronary atherosclerosis. A study showed an increased level of eotaxin, a specific chemokine of eosinophils in documented coronary artery disease patients (18). Another study reported an increase in eotaxin mRNA and protein levels in atherosclerotic plaques (19). However, the use of ECP as an eosinophil activation marker is increasing.

ECP is a highly cationic protein, which is a clinical marker of the activation of eosinophils released via various triggers, which are stored in peroxidase-positive and negative eosinophilic granules (20). The increases in the ECP level and it's correlation with disease severity have been demonstrated in autoimmune diseases such as asthma, atopic disorders, rheumatoid arthritis and psoriasis (21). ECP have also been shown in vascular necrotic/thrombotic lesions, which occur in temporal arthritis (22). One study showed that ECP increases the expression of ICAM-1, which induces the adhesion of monocytes to endothelium, thereby establishing an important step of atherogenesis and also joins in the activation of the coagulation cascade (23-25). Also, several studies revealed that ECP is involved in thrombus formation triggered by platelet activation and thrombomodulin inhibition in hypereosinophilic syndrome and allergic diseases (23-24).

A study by Niccoli et al (24) showed importantly higher serum ECP levels in patients with stable angina and non-ST elevation acute coronary syndrome relative to patients with normal coronary arteries. Again, some studies demonstrated a relation among ECP levels and the occurrence of cardiac events in patients undergoing implantation of first-production drug eluting stent and bare metal stents (26-28). Niccoci et al (4) study also showed that ECP has an association with in-stent restenosis, and neointimal burden. Rios-Navarro and et al (29) study showed that the ECP levels increased before reperfusion, were highest at $24 \mathrm{~h}$ and $96 \mathrm{~h}$, and returned to basal levels 1 month post-reperfusion in STEMI patients. In addition, their study reported that the eosinophil count markedly reduced soon after revascularization, this early activation and degranulation of eosinophils in acute myocardial infarction correlated with microvascular occlusion, edema, large infarct size, more extensive myocardial damage, and adverse cardiac events (30). Nicolli et al (30) study showed that serum levels of eosinophil cationic protein predicted major adverse cardiac events at 24 months in a population of patients with ST-segment elevation myocardial infarction treated with primary PCI, independently of baseline left ventricular ejection fraction. This predictive role of ECP might be explained by the higher thrombus score associated with raised eosinophil cationic protein levels. There are some suggestions that the treatments targeting eosinophils can be tested in ACS patients presenting with high ECP levels.

Despite the increasing number of studies with ECP and eosinophils, it has not been previously investigated in the phenomenon of CSF, where there are many mechanisms in its pathophysiology (27). Our study showed that eosinophil counts and ECP levels are increased and positively correlated in CSF patients. Our findings supported previous studies and were also important in terms of demonstrating higher ECP levels in CSF phenomenon (19-20). In addition, our findings showed that the ECP value was an indepen- 
dent predictor for the presence of CSF and may be used as suitable diagnostic biomarker for CSF. Based on this data, eosinophils and increased ECP levels may be a significant actor and marker of microvascular dysfunction and inflammatory process leading to CSF.

\section{Study limitations}

This study has several limitations. The main limitation of our study is the fact that a manual injector was used during coronary angiography. This may have affected the coronary flow rate. But, this was the case in both groups. The cross-sectional design of our study was unable to assess the long-time associations. Our study had limited number of patients (120). Lastly, prospective longterm follow-up studies with big data are required to enable the use routine as diagnostic and prognostic markers.

\section{Conclusion}

This is the first study to show that ECP levels are increased in CSF patients compared to normal coronary flow patients and this increase correlates with coronary artery flow rate parameters. The ECP level was independent predictor variable for the presence of CSF. In addition, ECP may be used as suitable diagnostic biomarker for CSF.

\section{References}

1. Horjeti B, Goda A. Acute ischemia manifestation in a patient with coronary slow flow phenomenon. J Electrocardiol 2012; 45: 277-279.

2. Acikel S, Bozkaya OA, Akdemir R. The relationship between intermittent left bundle-branch block and slow coronary flow in a patient presenting with acute coronary syndrome. Blood Coagul Fibrinolysis 2010; 21: 595-597.

3. Sasano H, Virmani R, Patterson RH et al. Eosinophilic products lead to myocardial damage. Hum Pathol 1989; 20: 850-857.

4. Niccoli G, Dato I, Imaeva AE et al. Association between inflammatory biomarkers and in-stent restenosis tissue features: an Optical Coherence Tomography Study. Eur Heart J Cardiovasc Imaging 2014; 15: 917-925.

5. Soylu K, Gulel O, Yucel $\mathbf{H}$ et al. The effect of blood cell count on coronary flow in patients with coronary slow phenomenon. Pak J Med Sci 2014; 30: 936-941.

6. Gibson CM, Cannon CP, Daley WL et al. TIMI frame count: a quantitative method of assessing coronary artery flow. Circulation 1996; 93: 879-888.

7. Isik T, Ayhan E, Uyarel $\mathbf{H}$ et al. Increased mean platelet volume associated with extent of slow coronary flow. Cardiol J 2012; 19: 355-362.

8. Dogan M, Akyel A, Cimen T et al. Relationship between Neutrophil to Lymphocyte Ratio and Slow Coronary Flow. Clin Appl Thromb Hemost 2015; 21: 251-254.

9. Li JJ, Qin XW, Li ZC et al. Increased plasma C-reactive protein and interleukin-6 concentrations in patients with slow coronary flow. Clin Chim Acta $2007 ; 385 ; 1-2: 43-47$.

10. Turhan H, Saydam GS, Erbay ER et al. Increased Plasma Soluble Adhesion Molecules; ICAM-1, VCAM-1, and E-selectin Levels in Patients with Slow Coronary Flow. Int J Cardiol 2006; 108; 2: 224-230.

11. Yavuz H, Chee R. A review on the vascular features of the hyper immunoglobulin E syndrome. Clin Exp Immunol 2010; 159: 238-244.

12. Umemoto S, Suzuki N, Fujii K et al. Eosinophil counts and plasma fibrinogen in patients with vasospastic angina pectoris. Am J Cardiol 2000; 85: 715-719.
13. Kounis NG. Coronary hypersensitivity disorder: the Kounis syndrome. Clin Ther 2013; 35: 563-571.

14. Nadimi AE, Ahmadi J, Mehrabian M. Peripheral eosinophil count and allergy in patients with coronary artery disease. Acta Med Indones 2008; 40: 74-77.

15. Sakai T, Inoue S, Matsuyama TA et al. Eosinophils may be involved in thrombus growth in acute coronary syndrome. Int Heart J 2009; 50: 267-277.

16. Rohrbach MS, Wheatley CL, Slifman NR et al. Activation of platelets by eosinophil granule proteins. J Exp Med 1990; 172: 1271-1274.

17. Samoszuk M, Corwin M, Hazen SL. Effects of human mast cell tryptase and eosinophil granule proteins on the kinetics of blood clotting. Am J Hematol 2003; 73: 18-25.

18. Economou E, Tousoulis D, Katinioti A et al. Chemokines in patients with ischaemic heart disease and the effect of coronary angioplasty. Int $\mathrm{J}$ Cardiol 2001; 80: 55-60.

19. Haley KJ, Lilly CM, Yang JH et al. Over expression of eotaxin and the CCR3 receptor in human atherosclerosis: using genomic technology to identify a potential novel pathway of vascular inflammation. Circulation 2000; 102: 2185-2189.

20. Venge P, Byström J, Carlson $\mathbf{M}$ et al. Eosinophil cationic protein (ECP): molecular and biological properties and the use of ECP as a marker of eosinophil activation in disease. Clin Exp Allergy 1999; 29: 1172-1186.

21. Hällgren R, Venge $\mathbf{P}$. The eosinophil in inflammation. In: Matsson $P$, Ahlstedt S, Venge P, Thorell J, editors. Clinical Impact of the Monitoring of Allergic Inflammation. London/San Diego: Academic Press 1991; 119-140.

22. Hällgren R, Gudbjörnsson B, Larsson E et al. Deposition of eosinophil cationic protein in vascular lesions in temporal arthritis. Ann Rheum Dis 1991; 50: 946-949.

23. Slungaard A, Vercellotti GM, Tran T et al. Eosinophil cationic granule proteins impair thrombomodulin function. A potential mechanism for thromboembolism in hyper eosinophilic heart disease. J Clin Invest 1993; 91: $1721-1730$.

24. Niccoli G, Ferrante G, Cosentino $\mathbf{N}$ et al. Eosinophil cationic protein: A new biomarker of coronary atherosclerosis. Atherosclerosis 2010; 211: 606-611.

25. Chihara J, Yamamoto T, Kurachi D et al. Possible release of eosinophil granule proteins in response to signaling from intercellular adhesion molecule-1 and its ligands. Int Arch Allergy Immunol 1995; 108 (Suppl 1): 52-54.

26. Niccoli G, Schiavino D, Belloni F, Ferrante G, La Torre G, Conte $\mathbf{M}$ et al. Preintervention eosinophil cationic protein serum levels predict clinical outcomes following implantation of drug-eluting stents. Eur Heart J 2009; 30: 1340-1347.

27. Niccoli G, Sgueglia GA, Conte M, Cosentino N, Minelli S, Belloni F et al. Eosinophil cationic protein and clinical outcome after bare metal stent implantation. Atherosclerosis 2011; 215: 166-9.

28. Yuan-Ting Zhu, Ling-Ping Zhu, Zhen-Yu Wang et al. Plasma Choline as a Diagnostic Biomarker in Slow Coronary Flow. Cardiol Res Pract 2020: 7361434 .

29. Rios-Navarro C, Gavara J, Vidal V et al. Characterization and implications of the dynamics of eosinophils in blood and in the infarcted myocardium after coronary reperfusion. PLoS One 2018; 13; 10: e0206344.

30. Niccoli G, Calvieri C, Flego D et al. Allergic Inflammation Is Associated With Coronary Instability and a Worse Clinical Outcome after Acute Myocardial Infarction. Circ Cardiovasc Interv 2015; 8; 8: e002554. 